\title{
The Impact of Opioid Treatment on Regional Gastrointestinal Transit
}

\author{
Jakob L Poulsen, ${ }^{1}$ Matias Nilsson, ${ }^{1}$ Christina Brock, ${ }^{1,2}$ Thomas H Sandberg, ${ }^{1}$ Klaus Krogh, ${ }^{3}$ and Asbjørn M Drewes ${ }^{1,4 *}$ \\ ${ }^{1}$ Mech-Sense, Department of Gastroenterology and Hepatology, Aalborg University Hospital, Aalborg, Denmark; ${ }^{2}$ Department of Drug \\ Design and Pharmacology, University of Copenhagen, Copenhagen, Denmark; ${ }^{3}$ Neurogastroenterology Unit, Department of Hepatology and \\ Gastroenterology, Aarhus University Hospital, Aarhus, Denmark; and ${ }^{4}$ Department of Clinical Medicine, Aalborg University, Aalborg, Denmark
}

\section{Background/Aims}

To employ an experimental model of opioid-induced bowel dysfunction in healthy human volunteers, and evaluate the impact of opioid treatment compared to placebo on gastrointestinal (GI) symptoms and motility assessed by questionnaires and regional $\mathrm{Gl}$ transit times using the 3-dimensional (3D)-Transit system.

\section{Methods}

Twenty-five healthy males were randomly assigned to oxycodone or placebo for 5 days in a double blind, crossover design. Adverse $\mathrm{Gl}$ effects were measured with the bowel function index, gastrointestinal symptom rating scale, patient assessment of constipation symptom questionnaire, and Bristol stool form scale. Regional GI transit times were determined using the 3D-Transit system, and segmental transit times in the colon were determined using a custom Matlab ${ }^{\circledR}$ graphical user interface.

\section{Results}

GI symptom scores increased significantly across all applied GI questionnaires during opioid treatment. Oxycodone increased median total Gl transit time from 22.2 to 43.9 hours $(P<0.001)$, segmental transit times in the cecum and ascending colon from 5.7 to 9.9 hours $(P=0.012)$, rectosigmoid colon transit from 2.7 to 9.0 hours $(P=0.044)$, and colorectal transit time from 18.6 to 38.6 hours $(P=0.001)$. No associations between questionnaire scores and segmental transit times were detected.

\section{Conclusions}

Self-assessed Gl adverse effects and increased GI transit times in different segments were induced during oxycodone treatment. This detailed information about segmental changes in motility has great potential for future interventional head-to-head trials of different laxative regimes for prevention and treatment of constipation.

(J Neurogastroenterol Motil 2016;22:282-291)

\section{Key Words}

Analgesics; Constipation; Gastrointestinal transit; Opioid

Received: October 27, 2015 Revised: December 22, 2015 Accepted: December 31, 2015

(a) This is an Open Access article distributed under the terms of the Creative Commons Attribution Non-Commercial License (http://creativecommons. org/licenses/by-nc/4.0) which permits unrestricted non-commercial use, distribution, and reproduction in any medium, provided the original work is properly cited.

${ }^{*}$ Correspondence: Asbjørn M Drewes, MD, PhD, DMSc

Mech-Sense, Department of Gastroenterology and Hepatology, Aalborg University Hospital, Mølleparkvej 4, DK-9000 Aalborg, Denmark

Tel: +45-9766-3562, Fax: +45-9766-3577, E-mail: amd@rn.dk 


\section{Introduction}

Gastrointestinal (GI) adverse effects are common in opioid treated patients and collectively referred to as opioid-induced bowel dysfunction (OIBD). ${ }^{1,2}$ They result from binding of exogenous opioids to opioid-receptors in the enteric nervous system, consequently disturbing normal GI function and manifesting in symptoms including gastroesophageal reflux, vomiting, bloating, abdominal pain, anorexia, hard stools, constipation, and incomplete evacuation. ${ }^{3}$ Severe adverse effects are often the reason patients discontinue opioid treatment, which naturally results in inadequate pain management. ${ }^{4,5}$ As the prescription of opioids has increased several fold in Europe and the US recent years, an increase in adverse effects to opioid treatment has expectedly also developed. ${ }^{6}$

Current assessments of OIBD typically rely on self-assessed questionnaires primarily focusing on constipation. ${ }^{7,8}$ The most commonly used questionnaires include assessment of number of spontaneous bowel movements (SBMs), the patient assessment of constipation symptoms (PAC-SYM) questionnaire, ${ }^{9}$ the bowel function index (BFI), ${ }^{10}$ and the Bristol stool form scale (BSFS). ${ }^{11}$ Despite elaborate focus on constipation, which is often defined as a reduction in number of SBMs, infrequent bowel movement ranks number 5 in self-assessed constipation symptoms, whereas symptoms such as gas, straining, and abdominal discomfort are far more prevalent. ${ }^{12}$ This finding strongly emphasizes that focusing on SBMs is inadequate to describe the complete burden of OIBD. Furthermore, other health-related risk factors such as reduced mobility, age, low-fiber diet, gender, and different drugs may also contribute to the collective GI symptomatology in opioid treated patients. ${ }^{13}$

The clinical presentation of OIBD is the result of decreased motility, decreased secretion, and dyscoordination of sphincter function in the GI tract. ${ }^{14}$ It has previously been shown, using invasive peroral cecal intubation and serial scintigrams, that subcutaneously administered morphine delays cecum and ascending colon transit and progression of the geometric center. ${ }^{15}$ However, reliable and minimally-invasive objective measures of OIBD are warranted in order to achieve a better understanding of the underlying mechanisms and evaluate the effect of novel pharmacological treatments on motility patterns. An attractive means of quantifying gut motility is the 3-dimensional (3D)-Transit system. This is a recently introduced, minimally-invasive, ambulatory system which—using a body-borne detection matrix - simultaneously tracks the precise position and general orientation of an electromagnetic capsule from ingestion to expulsion. ${ }^{16-19}$ Hence, the pan-enteric impact of opioid treatment can be accurately assessed, providing new insight into altered motility patterns. ${ }^{20}$ However, opioid treated patients often suffer from comorbidities and concomitant drug use, which complicates evaluation of the underlying pathophysiological mechanisms. Consequently, an experimental model in healthy volunteers, where these limitations are minimized, is highly merited.

It was hypothesized that an experimental model of OIBD in healthy human volunteers could be established through a 5-day opioid treatment. To test this hypothesis the aims were to evaluate how orally administered opioid treatment when compared to placebo affected (1) GI symptoms and function assessed by questionnaires, (2) regional and segmental GI transit times using the 3D-Transit system, and (3) investigate whether there was an association between self-assessed symptomatology and transit times.

\section{Materials and Methods}

The trial was approved by the local scientific ethical committee, the Danish Health and Medicines Authority, and conducted in accordance with the principles of ICH-GCP of the European Union. All subjects signed informed written consent according to the Declaration of Helsinki. The experiment was carried out between April 2014 and February 2015 at the research facilities of Mech-Sense, Aalborg University Hospital, Department of Gastroenterology \& Hepatology and Department of Radiology. The trial was registered at www.clinicaltrialsregister.eu (EudraCT number: 2013-001540$60)$.

\section{Subjects}

Twenty-five healthy, male volunteers (median age 24 years [21 to 56 years], mean height $182.2 \mathrm{~cm}$ [180 to $184.5 \mathrm{~cm}$ ], mean BMI $23.9 \mathrm{~kg} / \mathrm{m}^{2}$ [22.9 to $\left.25.0 \mathrm{~kg} / \mathrm{m}^{2}\right]$ ), with neither current symptoms nor history of GI disease were included in the study. All subjects underwent a screening session where the volunteers were informed about the study and informed consent was obtained. Hereafter, a physician (J.L.P) evaluated their medical history and ensured that all inclusion and exclusion criteria were fulfilled, and, if eligible, subjects were enrolled in the study.

Inclusion criteria were (1) signed written informed consent declaration, (2) capable of reading and understanding Danish, (3) male of Northern European descent, and (4) understanding of what the study involved: age 20-60 years, healthy, and opioid naive. The exclusion criteria were (1) known allergy towards opioids, (2) participation in any other studies within 14 days of enrolment, (3) 
planned medical/surgical treatment within the study duration, (4) a need to operate heavy machinery or motor vehicles during the study, (5) any previous or current drug abuse, (6) non-removable piercings or metal implants, (7) daily alcohol or nicotine consumption, (8) any known disease that may influence the results, and (9) the use of prescribed medicine and/or herbal medicine.

\section{Study Design}

Subjects were treated for 5 days (one period) with oral prolonged-release (PR) oxycodone or placebo in a double blind, crossover design (Fig. 1). A computer generated block-randomisation list ( 6 blocks of 4 subjects each and 1 subject manually randomised by study personnel which was not otherwise included in the study) was used. The personnel that generated the randomisation list also carried out proper concealment of each subject's assigned sequence in individually sealed envelopes, which allowed un-blinding of a single subject in case of emergencies. To ensure statistical power, dropouts were replaced using mirror-randomisation.

The subjects arrived at the research unit after an overnight fast. All periods started on a Monday (Day 1) where baseline GI questionnaires and muscle pressure stimulation thresholds were obtained. The first dose of PR oxycodone or placebo was administered to the subject after the baseline measurements and then repeated every 12 hours until Friday morning. Following the first

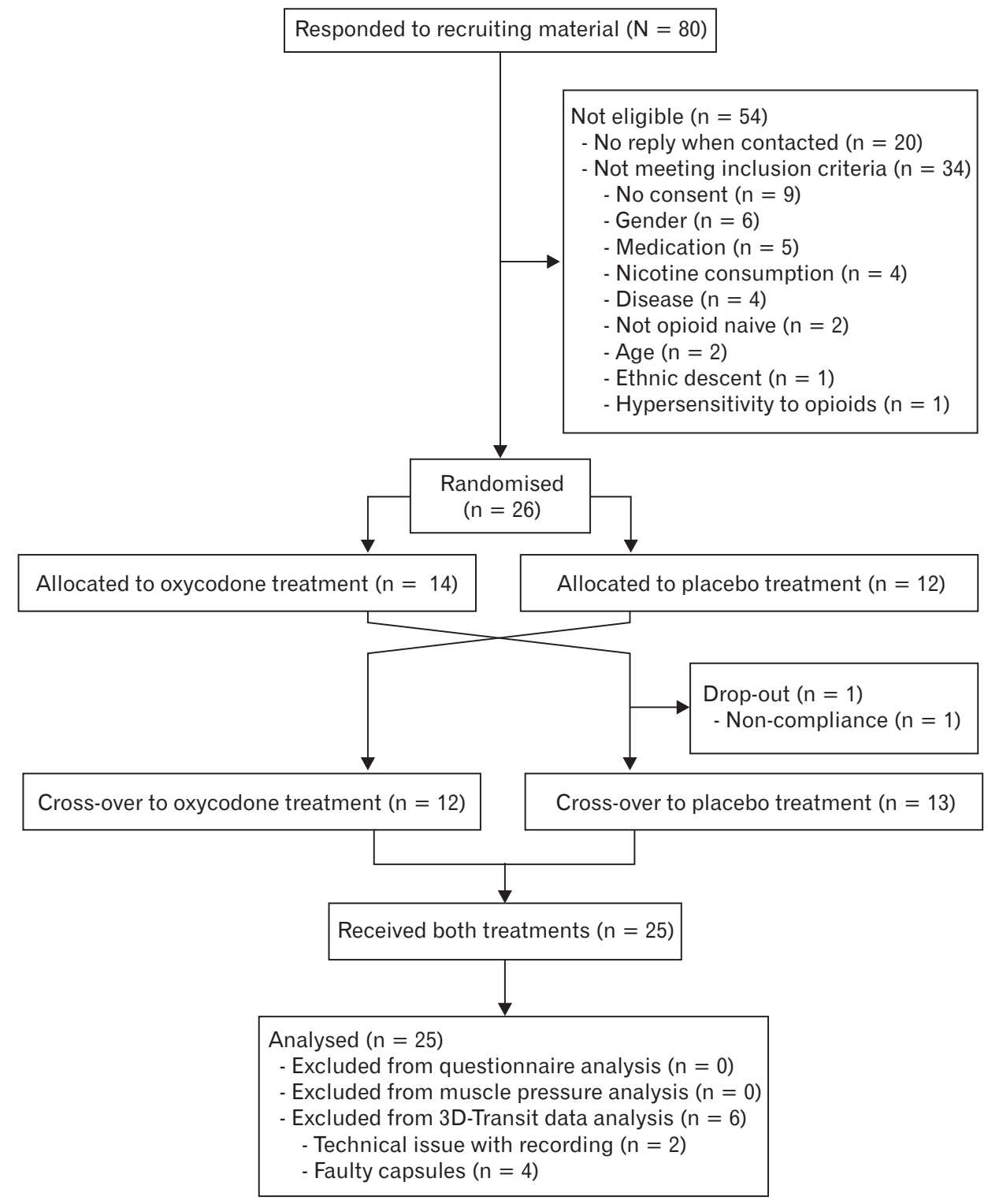

Figure 1. Study enrolment and randomisation. 
dose, a standardized meal (375 kcal, $11.4 \mathrm{~g}$ fat, and $1.8 \mathrm{~g}$ fiber) was ingested and immediately after the meal was finished, the 3DTransit capsule was ingested with a glass of water, approximately 15 minutes after the first dose of study medication. PR oxycodone was administered $5 \mathrm{mg}$ twice daily on Monday, $10 \mathrm{mg}$ twice daily on Tuesday through Thursday, and $10 \mathrm{mg}$ once Friday morning.

The data presented here are part of a larger protocol evaluating experimentally induced OIBD, which also included measures of colonic volume with magnetic resonance imaging (Nilsson et al. unpublished data).

\section{Questionnaires}

The subjects were asked to answer 4 validated GI questionnaires (1) BFI, ${ }^{10}(2)$ gastrointestinal symptom rating scale (GSRS), ${ }^{21}$ (3) PAC-SYM, ${ }^{9,22}$ and (4) BSFS. ${ }^{11}$ The BFI and GSRS were administered on day 1 and day 5 , whilst the PAC-SYM and BSFS were used to assess the dynamic changes of the general bowel function of the healthy volunteers and thus assessed daily.

\section{Muscle Pressure Stimulation}

To verify that the chosen oxycodone dose regimen was sufficient to provide analgesic effect, pressure algometry was included as a measure of analgesic effect. In order to improve consistency, stimuli were applied by the same examiner. ${ }^{23}$ Subjects were trained in assessment of pain using a modified visual analog scale (VAS; a continuous scale from 0-10 with anchor words for every increment of one). The scale has been described in details previously and used extensively to assess pain intensity in several different tissues. ${ }^{24-26}$ Pressure was applied on the dorsal forearm $10 \mathrm{~cm}$ distal to the elbow corresponding to the extensor digitorum muscle with a handheld algometer (Type 2; Somedic Production AB, Hörby, Sweden). The force increase rate was $30 \mathrm{kPa} / \mathrm{sec}$ adjusted to a probe size of 1 $\mathrm{cm}^{2}$. Two stimulations, separated by 10 seconds, were applied each day; corresponding to pain detection threshold and moderate pain (VAS $=5$ and VAS $=7$, respectively on the modified VAS Scale).

\section{D-Transit System}

The technical specifications, performance and use of the 3DTransit system is described in detail elsewhere. ${ }^{20}$ After ingestion of the 3D-Transit capsule, the subjects were instructed to fast for 6 hours as non-digestible solids are thought to empty with phase III of the migrating motor complex, and hence a new meal within 6 hours of capsule ingestion could delay gastric emptying. ${ }^{27}$ Subjects returned to the research facility on days $2,3,4$, and 5 , so that emitted signals from the capsules could be monitored in real-time.
This was done to confirm retention or expulsion of the capsules. Subjects were asked to refrain from hard physical work and sports. To confirm expulsion of the capsule by loss of signal on the 3DTransit recording, subjects were instructed to register the exact time of defecation during the study periods. Total GI transit time was defined as the time between ingestion and expulsion of the capsule. If the capsule was not expelled by day 5 , the recording was stopped, and the time of the last confirmed signal from the capsule was used as the earliest possible expulsion time. Based on previously validated principles, regional transit times were determined in the 3D-Transit analysis software from changes in contraction frequencies observed on the capsule rotation graphs, the time-frequency map for the recording, and 2D overview of capsule position. ${ }^{18}$ Gastric emptying was defined as the time from ingestion until pyloric passage, the latter identified as a shift from approximately 3 contractions per minute (cpm) to $12 \mathrm{cpm}$, in combination with the characteristic 2D image of passage through the duodenal arch. As the ingestion time for the first dose of oxycodone and ingestion time of the 3DTransit capsule was only 15 minutes apart, gastric emptying was not analysed in this study, as the short time interval did not allow for oxycodone to be sufficiently absorbed and induce OIBD in the gastric segment. Small intestinal transit time was defined as the time between pyloric passage and ileocecal passage. Ileocecal passage was identified as a decrease in contraction frequency from 6 cpm, characteristic of the distal ileum, to $3 \mathrm{cpm}$, typical of the colon. Identification of ileocecal passage was supported by a rapid lateral movement in the lower right quadrant. Total colorectal transit time was defined as the time between ileocecal passage and expulsion of the capsule.

In order to obtain the segmental transit times of the colon, a new method for 3D visualization and determination of the trajectory traversed by the magnetic capsule in the colon was developed. The raw positional data from the magnetic capsule were pre-processed prior to visualization. To enhance visualization of the trajectory, samples with considerable noise and artefacts coming from patient movement or external noise based on signal strength of the capsule and accelerometer data were removed. Additionally, the positional data were low-pass filtered using a second order Butterworth filter. Samples were then averaged for every 10 minutes providing a more uniform trajectory visualization as the capsule may remain in the same position within the colon for several hours.

The algorithms were incorporated into a custom Matlab (R2014b version 8.4.0.150421, MathWorks, Inc, Natick, MA, USA) application with a graphical user interface allowing the user to visually inspect the trajectory traversed by the capsule and select 
landmarks corresponding to the hepatic flexure, splenic flexure, and descending-sigmoid junction. Hereby, segmental colonic transit times could be calculated. In Figure 2, an example of a 3D trajectory visualized in the graphical user interface is shown.

All regional transit times were determined by the same investigator (J.L.P.). Inter-observer agreement was not done, as it previously has been shown to be good for both an earlier version of the 3D-Transit system ${ }^{18}$ and the same version as used in the present study. ${ }^{20}$ All segmental colonic transit times were determined by the same investigator (T.H.S.). In case of doubt, regional or segmental passage was discussed with another investigator (M.N., J.L.P., or T.H.S.).

\section{Data Analysis and Statistics}

All data are presented as means (95\% confidence interval) unless otherwise stated. All data were assessed for normality and handled accordingly with parametric or non-parametric statistics. Statistical analysis was performed with treatment coded as "treatment A" and "treatment B," and un-blinding was not performed until all data had been processed.

Baseline-corrected data from the BFI questionnaire were analysed with a paired $t$ test. Data from the GSRS questionnaire were

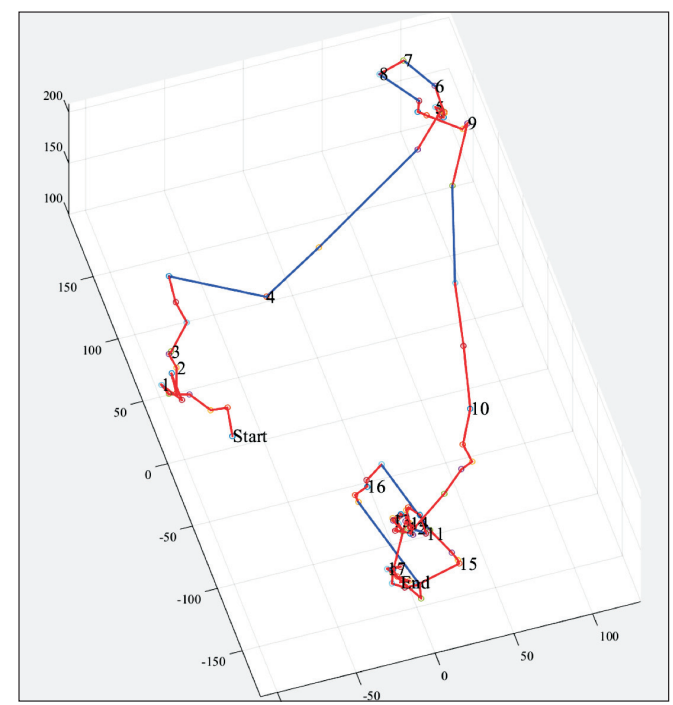

Figure 2. Example of 3-dimensional trajectory of colonic passage of a single capsule illustrated in the custom designed interactive graphical user interface. "Start" marks the ileocecal passage and "End" marks the expulsion of the capsule. A red line represents a progression of less than $50 \mathrm{~mm}$ the last 10 minutes. A blue line represents a progression of more than $50 \mathrm{~mm}$ during the last 10 minutes. Hepatic flexure, splenic flexure, and descending end can be marked in the trajectory in order to calculate segmental colonic transits. baseline-corrected and analysed in a two-way ANOVA with factors treatment (oxycodone and placebo) and questionnaire dimension (abdominal pain, reflux, indigestion, diarrhea, and constipation). Data from the PAC-SYM and BSFS questionnaires and pain detection thresholds were baseline-corrected and analysed with twoway ANOVAs with factors treatment (oxycodone and placebo) and day (days 2, 3, 4, and 5). For all ANOVAs, Tukey's post hoc test was applied where relevant. Transit time data were non-parametric, and are presented as medians and ranges. Wilcoxon signed rank test was used to compare segmental transit times during the 2 treatments. Spearman's rho was used to identify associations between questionnaire scores and transit times. $P$-values $<0.05$ were considered significant. Statistical analyses were carried out using Stata (Version 13.1; StataCorp LP, College Station, TX, USA) and SPSS (Version 22; release 22.0.0.0, IBM Corp, New York, USA).

\section{Results}

Twenty-six subjects were enrolled in the study; 1 subject was excluded during day 4 in the first period due to non-compliance. This allowed a total of 25 subjects to complete the study.

\section{Questionnaires}

BFI scores increased 34 points during oxycodone treatment vs 3 points increase during placebo treatment $(P<0.001)$ (Fig. 3). Total GSRS scores likewise increased during oxycodone treatment $(P<0.001)$ (Fig. 3). The post hoc test revealed increased scores during oxycodone treatment for the dimensions abdominal pain $(P$ $=0.031)$ and constipation $(P<0.001)$. For the PAC-SYM significantly higher scores were found during oxycodone treatment on day 4 (6.5 vs 1.9 during oxycodone vs placebo, respectively; $P<$ $0.001)$ and day 5 (7.0 vs 2.0 during oxycodone vs placebo, respectively; $P<0.001$ ), but also within the oxycodone treatment period there were significant increases between day 2 and day 4 ( 2.0 vs 6.5 day 2 vs day 4, respectively; $P=0.002$ ) and between day 2 and day 5 (2.0 vs 7.0 day 2 vs day 5 , respectively; $P<0.001$ ). Number of bowel movements were lower on day 2 during oxycodone treatment compared to placebo, 0.9 vs $1.7(P=0.001)$, but not for other days. Stool form scores also decreased significantly during oxycodone treatment for all days compared to the placebo $(37 \%, 52 \%$, $64 \%, 40 \%$, and $73 \%$ lower scores for day 1 through 5 ; all $P<0.05$ ).

\section{Muscle Pressure Stimulation}

The analgesic effect of oxycodone was validated in the pain model with an $8 \%$ increase in pain detection threshold to muscle 

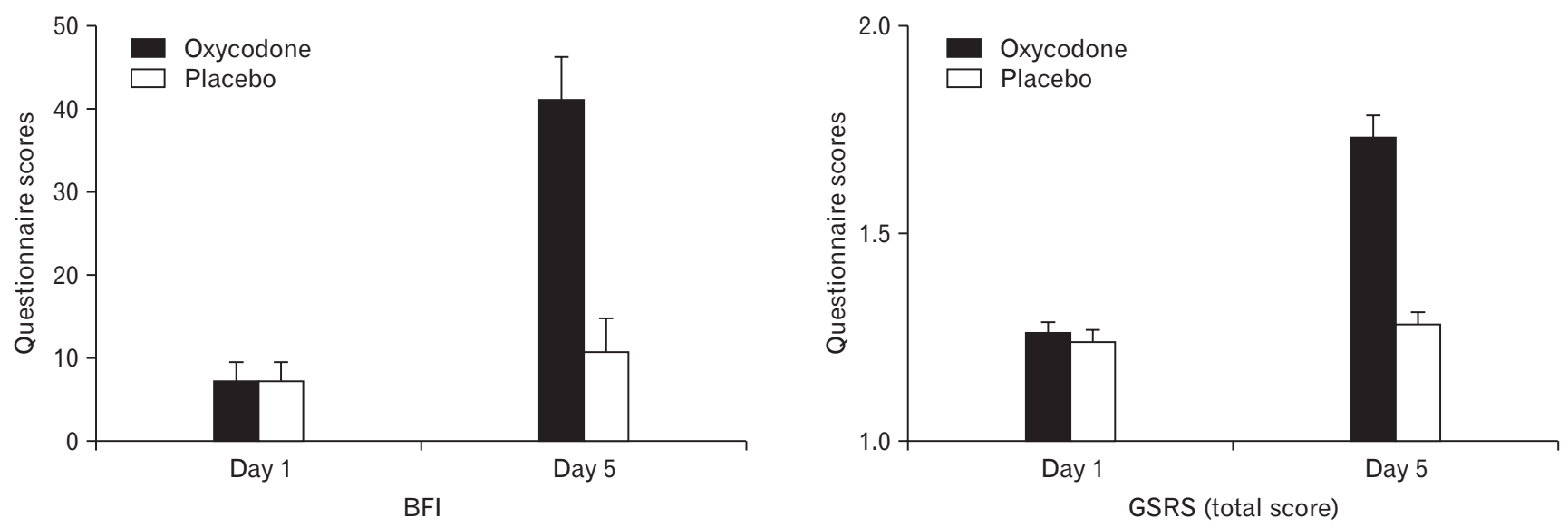

Figure 3. Bowel function index (BFI) and total gastrointestinal symptom rating scale (GSRS) results. From the left the first figure depicts the average BFI scores from day 1 and day 5 for both treatments. The second figure shows the total GSRS. Data are presented as means \pm SEM.

Table. Total and Segmental Transit Times (in Hours) During Placebo and Oxycodone Treatment

\begin{tabular}{lccc}
\hline & Placebo & Oxycodone & $P$-value \\
\hline Small intestinal transit time & $3.7(1.9$ to 10.0$)$ & $5.3(1.5$ to 9.6$)$ & 0.147 \\
Cecum and ascending colon & $5.7(0.4$ to 21.7$)$ & $9.9(0.3$ to 73.2$)$ & 0.012 \\
Transverse colon & $4.4(0.5$ to 40.6$)$ & $3.8(0.8$ to 4.4$)$ & 0.872 \\
Descending colon & $1.4(0.4$ to 15.6$)$ & $9.7(0.5$ to 25.8$)$ & 0.064 \\
Rectosigmoid colon & $2.7(0.1$ to 19.0$)$ & $38.6(5.6$ to 88.6$)$ & 0.044 \\
Colorectal transit time & $18.6(7.0$ to 82.2$)$ & $43.9(7.1$ to 92.6$)$ & 0.001 \\
Total GI transit time & $22.2(11.0$ to 85.6$)$ & & $<0.01$ \\
\hline
\end{tabular}

Data are presented as medians (range).

pressure in comparison to $3 \%$ decrease during placebo treatment $(P$ $=0.021)$.

\section{Total and Regional Transit Times}

Of the 50 capsules ingested ( 1 per period per subject), full recordings were obtained in 44 cases. Technical problems lead to insufficient recordings in 2 cases, in whom it was only possible to determine the total GI transit. In 4 cases it was not possible to determine any transits due to faulty capsules. This led to 19 subjects with full recordings of all regional and colon segment transits in both periods, and 21 subjects with total GI transit in both periods. Capsule retention on day 5 occurred in 7 recordings ( 6 during oxycodone treatment and 1 during placebo). No adverse events were registered.

Oxycodone treatment increased total GI transit $(P<0.001)$ and colorectal transit $(P=0.001)$. For the colonic segments, oxycodone treatment increased cecum and ascending colon transit time $(P=0.012)$ and rectosigmoid transit time $(P=0.044)$. No other segmental transits were significantly different during oxycodone treatment compared to placebo (Table).

\section{Correlations}

No associations were found between questionnaire scores and segmental transit times (all Spearman's rho $< \pm 0.5$; all $P>0.05$ ).

\section{Discussion}

This study has shown that the chosen dose regimen induced OIBD symptoms verified by increase in subjective symptom scores across all GI questionnaires. Furthermore, short term PR oxycodone treatment significantly increased the total GI transit time, colorectal transit time, and segmental colonic transit times in the cecum and ascending colon, as well as the rectosigmoid colon. The increase in pain detection thresholds suggests adherence to treatment. No associations between questionnaire scores and transit times were detected.

Both the BFI, specifically designed and validated to assess opioid-induced constipation, and the more comprehensive GSRS, 
increased significantly during oxycodone treatment, ${ }^{10}$ convincingly implying the desired constipating effect. Particularly interesting was that not only the constipation dimension of the GSRS but also the abdominal pain dimension increased. Of special note are the daily assessed PAC-SYM scores, revealing a progressively constipating effect with significant increases on day 4 and 5 -both during oxycodone treatment when compared to placebo, but also within the oxycodone arm when compared to day 2. In agreement, stool form score decreased for all days during oxycodone treatment compared to placebo, but SBM frequency only decreased on day 2 in the oxycodone arm compared to placebo.

The 3D-Transit system added interesting insights in regional transit times during oxycodone treatment. It has previously been shown that intravenous morphine increases oro-cecal transit evaluated by breath tests. ${ }^{28}$ The current data is supported by a comprehensive study by Kaufman et al, ${ }^{15}$ who showed that subcutaneously administered morphine decreases transit in the cecum and ascending colon as well as geometric center transit. However, fewer subjects underwent a highly invasive method with peroral cecal intubation in this study, where only fluid meals were allowed, and the subjects were subjected to a series of scintigrapic images, consequently exposing them for radiation. Thus, clinical studies investigating colonic transit in response to for example, pharmacological interventions, necessitate less invasive methods such as the ambulatory and minimally-invasive nature of the 3D-Transit system.

The significantly prolonged transit during opioid treatment in the cecum and ascending colon, but not in the transverse and descending colon, indicates that the origin of pathophysiological motility is in this colonic segment. The analysis software for the 3DTransit system is still in its infancy, but the current system is able to provide precise anatomical position of ingested capsules, description of contractile activity and luminal progression through well-defined regions of the GI tract. ${ }^{20}$ An alternative, but invasive, method to examine colonic motility patterns is high-resolution fiber-optic manometry. ${ }^{29} \mathrm{~A}$ recent study employing this technique in slow transit constipation patients found that the normal increase in the postprandial propagating cyclic motor pattern was absent in patients when compared to healthy controls. ${ }^{30}$ Similar studies in slow transit constipation patients have found a reduced daily frequency of high amplitude propagating sequences (also recognised as mass movements and responsible for a significant proportion of propulsive activity in the colon), and that all high amplitude propagating sequences originate in the proximal colon. ${ }^{29,31,32}$ It is therefore plausible that the prolonged colonic transit times during oxycodone treatment in this study share—at least partly—pathophysiological characteristics with slow transit constipation, and that further development of the 3D-Transit software could elucidate this neglected, yet important aspect of GI motility. Along the same line, the increased transit in the rectosigmoid colon during oxycodone treatment could indicate that not only transit, but possibly also defecation, is affected. This may be an indirect consequence of prolonged transit leading to increased passive absorption, resulting in harder, dryer stools, which are difficult to pass. In addition, the recto-anal inhibitory reflex has also been found to be inhibited during opioid administration further complicating defecation. ${ }^{33}$ No difference in small intestinal transit was observed, but as previous studies have been contradictory, methodological refinements are likely needed to report consistent findings. ${ }^{34-36}$ Recent in vitro studies in rodents has demonstrated tolerance to opioids in the upper, but not the lower GI tract. ${ }^{37,38}$ Therefore differences in tolerance may partly explain our findings although direct translation to human in vivo physiology is highly questionable. $^{39}$

No associations between questionnaire scores and transit times were found. At first, this may seem surprising, but could reflect that the clinical presentation of OIBD is multi-faceted and the self-assessed symptoms are highly subjective, and does not always correspond with the objective findings. This is underscored in a study by Bell et al, ${ }^{40}$ who found that many opioid-treated patients report normal stool frequency, but still experience symptoms of OIBD, and Johanson and Kralstein ${ }^{12}$ who found that infrequent bowel movement ranks only number 5 in self-assessed constipation symptoms. Moreover, stool frequency correlates poorly with both measured whole-gut and colonic transit time, and only a modest correlation between GI transit and stool form exists in chronic constipated patients. ${ }^{41}$ This strongly accentuates the limitations of studies using number of SBMs as the primary outcome measure, as it may potentially underestimate symptoms regarded more bothersome for the patient. Our study supports these results, as SBM frequency only changed on day 2, but other OIBD symptoms were present throughout the study along with the significant increased transit times in the colon.

A major advantage of our study is the use of healthy volunteers. In a clinical setting, factors known to affect bowel function such as immobility, concomitant drug use, dehydration, anorexia, previous GI surgery, haemorrhoids, but also psychological factors such as depression and anxiety can influence the outcome, and hence it can be difficult to evaluate the contribution of the variable of interestin this case oxycodone. ${ }^{42}$ By applying an experimental model, where these factors are controlled, this allows us to examine the underlying pathophysiology and development of GI symptoms during oxyco- 
done treatment with low risk of confounding and bias. Of course, a model like this should be validated in a clinical setting. Due to ethical reasons we chose only to treat the volunteers for 5 days. Despite this, our results clearly show that significant changes in bowel function and GI adverse effects could be induced. It is therefore plausible, that a longer treatment and/or higher dose would have resulted in even more pronounced symptoms. This scenario would also be more similar to the typical opioid treated patient. Moreover, a chronic pain condition may also result in a more sedentary lifestyle, known to negatively affect bowel function. ${ }^{43}$

The lack of gastric emptying data could influence the transit time and upper gut function during oxycodone treatment, but due to the logistics in study design these were not valid. Furthermore, the loss of data due to technical problems and faulty capsules resulting in 19 subjects with full recordings for all GI regions was unfortunate, as this, combined with the wide intra- and inter-individual variation in transit times, hampers the possibility to detect significant differences. Capsule retention on day 5 in 7 recordings affected precise calculations of colorectal and total GI transit times. As it occurred in 6 recordings during oxycodone treatment, but only once in the placebo period, it has resulted in a systematic underestimation of colorectal and total GI transit time during oxycodone treatment. Also, one could argue that the use of only male volunteers in our study restricts external validity. The reason for this was to avoid potential confounding on GI symptoms and function due to the female menstrual cycle. ${ }^{4+46}$ As functional constipation is also more prevalent among women than men ${ }^{47}$ and if opioid-induced constipation and (slow-transit) functional constipation share pathophysiological mechanisms, it is not unlikely that the prevalence of GI adverse effects due to opioid treatment would be equally frequent in women.

In conclusion, OIBD can be experimentally induced through 5 days of oral PR oxycodone treatment, as subjectively evaluated by validated GI questionnaires and objectively by increased total GI transit time, colorectal transit time, and segmental colonic transit times in the cecum and ascending colon, and rectosigmoid colon. Our study highlights the advantage of combined, comprehensive subjective and objective evaluation, and the limitation of focusing on SBMs as the sole or primary outcome measure when assessing OIBD. Accordingly, this experimental model provides a tool for detailed investigation of pathophysiological motility in a controlled environment, which may be used in comparative studies of treatments against this burdensome condition.
Financial support: This study was funded (1) in part by Innovation Fund Denmark-Individuals, Disease and Society (Grant No. 10-092786), (2) in part by The A.P. Møller Foundation for the Advancement of Medical Science (Grant No. 14-319), (3) in part by Aage og Johanne Louis-Hansens Fond, and (4) in part by an unrestricted grant from Mundipharma Research GmbH \& Co. KG.

Conflicts of interest: Asbjørn M Drewes has received financial support from Mundipharma, AstraZeneca, Shire, Almirall, Grünenthal, and Pfizer. Klaus Krogh has served as an advisory board member for Coloplast, Wellspect, Nordic Health Care, Amirall, and Shire.

Author contributions: Jakob L Poulsen: data acquisition, data interpretation, drafting of manuscript, and statistical analysis; Matias Nilsson: data acquisition, data interpretation, and drafting of manuscript; Christina Brock: study supervision, study design, data interpretation, statistical analysis, and revision of the manuscript for important intellectual content; Thomas H Sandberg: wrote algorithm and graphical user interface for assessment of regional colonic transit time, data interpretation, and drafting of manuscript; Klaus Krogh: data interpretation and revision of the manuscript for important intellectual content; Asbjørn M Drewes: obtained funding for the study, study supervision, study design, data interpretation, and revision of the manuscript for important intellectual content.

\section{References}

1. De Schepper HU, Cremonini F, Park MI, Camilleri M. Opioids and the gut: pharmacology and current clinical experience. Neurogastroenterol Motil 2004;16:383-394.

2. Wood JD, Galligan JJ. Function of opioids in the enteric nervous system. Neurogastroenterol Motil 2004;16(suppl 2):17-28.

3. Pappagallo M. Incidence, prevalence, and management of opioid bowel dysfunction. Am J Surg 2001(5A suppl);182:11S-18S.

4. Thorpe DM. Management of opioid-induced constipation. Curr Pain Headache Rep 2001;5:237-240.

5. Tamayo AC, Diaz-Zuluaga PA. Management of opioid-induced bowel dysfunction in cancer patients. Support Care Cancer 2004;12:613-618.

6. Casati A, Sedefov R, Pfeiffer-Gerschel T. Misuse of medicines in the European union: a systematic review of the literature. Eur Addict Res 2012;18:228-245.

7. Olesen AE, Drewes AM. Validated tools for evaluating opioid-induced bowel dysfunction. Adv Ther 2011;28:279-294.

8. Gaertner J, Siemens W, Camilleri M, et al. Definitions and outcome measures of clinical trials regarding opioid-induced constipation: a systematic review. J Clin Gastroenterol 2015;49:9-16. 
9. Slappendel R, Simpson K, Dubois D, Keininger DL. Validation of the PAC-SYM questionnaire for opioid-induced constipation in patients with chronic low back pain. Eur J Pain 2006;10:209-217.

10. Rentz AM, Yu R, Müller-Lissner S, Leyendecker P. Validation of the bowel function index to detect clinically meaningful changes in opioidinduced constipation. J Med Econ 2009;12:371-383.

11. Lewis SJ, Heaton KW. Stool form scale as a useful guide to intestinal transit time. Scand J Gastroenterol 1997;32:920-924.

12. Johanson JF, Kralstein J. Chronic constipation: a survey of the patient perspective. Aliment Pharmacol Ther 2007;25:599-608.

13. Wirz S, Nadstawek J, Elsen C, Junker U, Wartenberg HC. Laxative management in ambulatory cancer patients on opioid therapy: a prospective, open-label investigation of polyethylene glycol, sodium picosulphate and lactulose. Eur J Cancer Care (Engl) 2012;21:131-140.

14. Brock C, Olesen SS, Olesen AE, Frøkjaer JB, Andresen T, Drewes AM. Opioid-induced bowel dysfunction: pathophysiology and management. Drugs 2012;72:1847-1865.

15. Kaufman PN, Krevsky B, Malmud LS, et al. Role of opiate receptors in the regulation of colonic transit. Gastroenterology 1988;94:1351-1356.

16. Stathopoulos E, Schlageter V, Meyrat B, De Ribaupierre Y, Kucera P. Magnetic pill tracking: a novel non-invasive tool for investigation of human digestive motility. Neurogastroenterol Motil 2005;17:148-154

17. Hiroz P, Schlageter V, Givel JC, Kucera P. Colonic movements in healthy subjects as monitored by a magnet tracking system. Neurogastroenterol Motil 2009;21:838, e57.

18. Worsøe J, Fynne L, Gregersen T, et al. Gastric transit and small intestinal transit time and motility assessed by a magnet tracking system. BMC Gastroenterol 2011;11:145.

19. Hedsund C, Joensson IM, Gregersen T, Fynne L, Schlageter V, Krogh $\mathrm{K}$. Magnet tracking allows assessment of regional gastrointestinal transit times in children. Clin Exp Gastroenterol 2013;6:201-208.

20. Haase AM, Gregersen T, Schlageter V, et al. Pilot study trialling a new ambulatory method for the clinical assessment of regional gastrointestinal transit using multiple electromagnetic capsules. Neurogastroenterol Motil 2014;26:1783-1791.

21. Kulich KR, Madisch A, Pacini F, et al. Reliability and validity of the gastrointestinal symptom rating scale (GSRS) and quality of life in reflux and dyspepsia (QOLRAD) questionnaire in dyspepsia: a six-country study. Health Qual Life Outcomes 2008;6:12.

22. Frank L, Kleinman L, Farup C, Taylor L, Miner P Jr. Psychometric validation of a constipation symptom assessment questionnaire. Scand $\mathrm{J}$ Gastroenterol 1999;34:870-877.

23. Modir JG, Wallace MS. Human experimental pain models 3: heat/capsaicin sensitization and intradermal capsaicin models. Methods Mol Biol 2010;617:169-174.

24. Andresen T, Staahl C, Oksche A, Mansikka H, Arendt-Nielsen L, Drewes AM. Effect of buprenorphine and fentanyl in experimental induced superficial, deep and hyperalgesic pain. Br J Pharmacol 2011;164:934-945.

25. Drewes AM, Gregersen H, Arendt-Nielsen L. Experimental pain in gastroenterology: a reappraisal of human studies. Scand J Gastroenterol 2003;38:1115-1130.
26. Staahl C, Christrup LL, Andersen SD, Arendt-Nielsen L, Drewes AM. A comparative study of oxycodone and morphine in a multi-modal, tissue-differentiated experimental pain model. Pain 2006;123:28-36.

27. Cassilly D, Kantor S, Knight LC, et al. Gastric emptying of a nondigestible solid: assessment with simultaneous SmartPill $\mathrm{pH}$ and pressure capsule, antroduodenal manometry, gastric emptying scintigraphy. Neurogastroenterol Motil 2008;20:311-319.

28. Yuan CS, Foss JF, Osinski J, Toledano A, Roizen MF, Moss J. The safety and efficacy of oral methylnaltrexone in preventing morphine-induced delay in oral-cecal transit time. Clin Pharmacol Ther 1997;61:467-475.

29. Dinning PG, Wiklendt L, Maslen L, et al. Quantification of in vivo colonic motor patterns in healthy humans before and after a meal revealed by high-resolution fiber-optic manometry. Neurogastroenterol Motil 2014;26:1443-1457.

30. Dinning PG, Wiklendt L, Maslen L, et al. Colonic motor abnormalities in slow transit constipation defined by high resolution, fibre-optic manometry. Neurogastroenterol Motil 2015;27:379-388.

31. Rao SS, Sadeghi P, Beaty J, Kavlock R. Ambulatory 24-hour colonic manometry in slow-transit constipation. Am J Gastroenterol 2004;99:24052416.

32. Bassotti G, Gaburri M, Imbimbo BP, et al. Colonic mass movements in idiopathic chronic constipation. Gut 1988;29:1173-1179.

33. Musial F, Enck P, Kalveram KT, Erckenbrecht JF. The effect of loperamide on anorectal function in normal healthy men. J Clin Gastroenterol 1992;15:321-324.

34. Jeong ID, Camilleri M, Shin A, et al. A randomised, placebo-controlled trial comparing the effects of tapentadol and oxycodone on gastrointestinal and colonic transit in healthy humans. Aliment Pharmacol Ther 2012;35:1088-1096

35. Maurer AH, Krevsky B, Knight LC, Brown K. Opioid and opioid-like drug effects on whole-gut transit measured by scintigraphy. J Nucl Med 1996;37:818-822.

36. Yee MK, Evans WD, Facey PE, Hayward MW, Rosen M. Gastric emptying and small bowel transit in male volunteers after i.m. ketorolac and morphine. Br J Anaesth 1991;67:426-431.

37. Akbarali HI, Inkisar A, Dewey WL. Site and mechanism of morphine tolerance in the gastrointestinal tract. Neurogastroenterol Motil 2014;26:1361-1367.

38. Kang M, Maguma HT, Smith TH, Ross GR, Dewey WL, Akbarali $\mathrm{HI}$. The role of $\beta$-arrestin 2 in the mechanism of morphine tolerance in the mouse and guinea pig gastrointestinal tract. J Pharmacol Exp Ther 2012;340:567-576.

39. De Luca A, Coupar IM. Insights into opioid action in the intestinal tract. Pharmacol Ther 1996;69:103-115.

40. Bell TJ, Panchal SJ, Miaskowski C, Bolge SC, Milanova T, Williamson $\mathrm{R}$. The prevalence, severity, and impact of opioid-induced bowel dysfunction: results of a US and European Patient Survey (PROBE 1). Pain Med 2009;10:35-42.

41. Saad RJ, Rao SSC, Koch KL, et al. Do stool form and frequency correlate with whole-gut and colonic transit? Results from a multicenter study in constipated individuals and healthy controls. Am J Gastroenterol 2010;105:403-411. 
42. Porcelli P, Todarello O. Psychological factors affecting functional gastrointestinal disorders. Adv Psychosom Med 2007;28:34-56.

43. Dorn S, Lembo A, Cremonini F. Opioid-Induced bowel dysfunction: epidemiology, pathophysiology, diagnosis, and initial therapeutic approach. Am J Gastroenterol 2014;2:31-37.

44. Moore J, Barlow D, Jewell D, Kennedy S. Do gastrointestinal symptoms vary with the menstrual cycle? Br J Obstet Gynaecol 1998;105:13221325.

45. Heitkemper MM, Jarrett M. Pattern of gastrointestinal and somatic symptoms across the menstrual cycle. Gastroenterology 1992;102:505513.

46. Meier R, Beglinger C, Dederding JP, et al. Influence of age, gender, hormonal status and smoking habits on colonic transit time. Neurogastroenterol Motil 1995; 7:235-238.

47. Sandler RS, Jordan MC, Shelton BJ. Demographic and dietary determinants of constipation in the US population. Am J Public Health 1990;80:185-189. 